\title{
Umweltverschmutzung
}

\section{Weckt Feinstaub „schlafende“ Viren?}

Feinstaub soll Allergien verstärken, Asthmaanfälle auslösen und steht unter dem Verdacht, das

Herzinfarktrisiko zu erhöhen. Forscher vom Münchner Helmholtz Zentrum zeigten nun am Maus-

modell, dass Kohlenstoffnanopartikel auch latente Virusinfekte in der Lunge reaktivieren können.

PneumoNews: Herr Dr. Stöger, Sie und Ihr Team haben herausgefunden, dass in Feinstaub enthaltene Nanopartikel in der Lage sind, "schlafende“ Viren in der Lunge zu aktivieren. Wie ist das gelungen? Stöger: Wir wissen, dass Gamma-Herpesviren in bestimmten Zellen, z.B. B-Lymphozyten, aber auch Epithelzellen oder Makrophagen, latent persistieren können. So tragen in unseren Breiten über $95 \%$ aller Personen ab 40 Jahren „schlafende“ EpsteinBarr-Viren in sich, auch wenn nur die wenigsten bei ihrer Erstinfektion ein Pfeiffersches Drüsenfieber erlebt haben. Stress kann diese Latenz aufbrechen und zu einer Reaktivierung der Viren führen. Dies konnten wir gemeinsam mit dem virologischen Team von Prof. Heiko Adler zeigen, indem wir bei latent mit Herpesviren infizierten Mäusen Kohlenstoffnanopartikel oder auch -fasern im Alveolarbereich deponiert haben. Für die Zellen stellt das einen Stress-Stimulus dar. In früheren Untersuchungen kam es dadurch - abhängig von der Partikelform - für bestimmte Fasern zu relativ lang anhaltenden entzündlichen Reaktionen. Bei den mit Partikeln behandelten Mäusen konnten wir nun noch 28 Tage nach der Infektion virale lytische Proteine in der Lunge nachweisen. Das war für uns ein Hinweis dafür, dass es zu einer Art Reaktivierung des Herpesvirus gekommen sein musste. Die lytischen Proteine, die der Zerstörung der Wirtszelle und damit der Virusausbreitung dienen, mussten neu produziert worden sein, weil sie ja nicht so lange im Körper verweilen können.

PneumoNews: Welche Mechanismen vermuten Sie dahinter? Stöger: Wir vermuten, dass in den Zellen, die das Virus tragen, Programme unterdrückt werden, die normalerweise dafür sorgen, dass die infizierte Zelle vom Immunsystem erkannt wird.

PneumoNews: Ist das Modell auf den Menschen übertragbar? Stöger: Wir haben humane lymphoblastoide Zellen untersucht, die latentes Epstein-Barr-Virus tragen. Auch dieses hat sich in Zellkulturen reaktivieren lassen.

PneumoNews: Müssten, wenn Ihre Hypothese zutrifft, nicht viel mehr Menschen an Infektionen durch reaktivierte Viren erkranken?

Stöger: Das größte Fragezeichen ist die Feinstaubdosis, bei der das passiert. In dem Proof-of-Concept-Versuch haben wir Kohlenstoffnanopartikel als Suspension in die Atemwege gespült. Dabei haben wir relativ hohe Dosen verwendet und gefunden, dass eine Virusreaktivierung erfolgt. Die Dosen entsprechen etwa dem, was man an einem Arbeitsplatz mit Feinstaubkonzentrationen im zulässigen Maximalbereich ${ }^{*}$ in einer Woche akkumulieren könnte.

PneumoNews: Wie groß ist die Gefahr durch Feinstaub in Städten?

* TGRS 900 Arbeitsplatzgrenzwerte

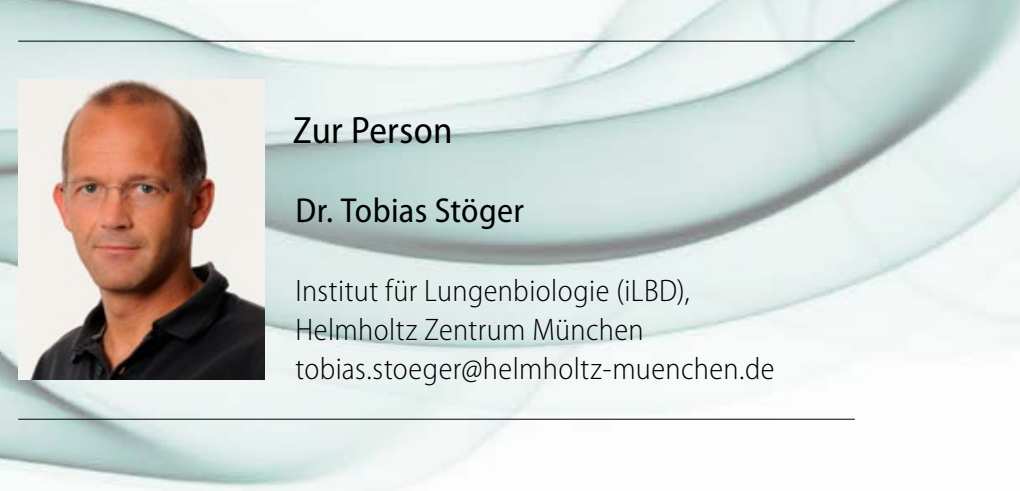

Stöger: Ob schon geringere Dosen, wie wir sie in Großstädten täglich erleben, zu einer messbaren Reaktivierung führen können, müssen wir noch prüfen. Wir haben ja bisher nur die akute Gabe untersucht. Was wir viel eher für bedenklich halten, sind die kleinen Dosen, die wir immer wieder abbekommen. Dies führt vielleicht nur zu einer geringgradigen Viren-Reaktivierung; man liegt nicht gleich darnieder, wie bei einer Grippe, aber es werden ständig Reaktivierungsprogramme induziert. Wir wollen nun untersuchen, ob das zu einer chronischen Belastung und zu einer anhaltenden Schädigung oder gar Lungenfibrose führen könnte.

\section{PneumoNews: Welche Personen sind am ehesten gefährdet?}

Stöger: Zum einen Personen mit hoher Feinstaubexposition. Auch jene mit Vorschädigungen der Lunge, Asthma, chronischobstruktiver oder granulomatöser Lungenerkrankung, könnten anfällig für eine Virusreaktivierung sein. Bisher war das Herzinfarktrisiko das, wovor wir am meisten Angst hatten. Die drohende Entwicklung von fibrotischen Veränderungen könnte hinzukommen. Man hat beobachtet, ohne es kausal begründen zu können, dass sich bei Lungenfibrosepatienten besonders viele Gamma-Herpesvirus-Infektionen finden, mit besonders hohem Virustiter. Das kann daher kommen, dass durch die Fibrose das Immunsystem geschwächt ist, oder daher, dass die verstärkte Virusreaktivierung bei den Patienten zu mehr fibrotischen Veränderungen führt. Man weiß also nicht, was Henne ist und was Ei.

PneumoNews: Wie soll der Arzt mit diesen Erkenntnissen umgehen? Stöger: Wir haben ja nur eine Tierstudie; das ist für sich allein noch kein Grund für zusätzliche Besorgnis. Fest steht: Feinstaub ist ungesund und man sollte sich nicht unnötig exponieren. Es kommen immer mehrere Faktoren zusammen. Der einzelne ist wohl nicht toxisch genug, aber die Summe kann dazu führen, dass gerade schwächere Menschen Symptome entwickeln. 